\title{
Quantitative Relief Models of Rock Surfaces on Mars at Sub-millimeter Scales from Mars Curiosity Rover Mars Hand Lens Imager (MAHLI) Observations: Geologic Implications
}

James B. Garvin ${ }^{1}$, Kenneth S. Edgett ${ }^{2}$, Ryan Dotson ${ }^{3,1}$, Deirdra M. Fey ${ }^{2}$, Kenneth E. Herkenhoff ${ }^{4}$, Bernard J. Hallet ${ }^{5}$, and Megan R. Kennedy ${ }^{2}$

1. NASA Goddard Space Flight Center (GSFC), Mail Code 600, Greenbelt, MD 20771, USA.

2. Malin Space Science Systems (MSSS), San Diego, CA, USA.

3. Fireball LLC, Reno, NV, USA (supporting NASA).

4. U.S. Geological Survey, Astrogeology Science Center, Flagstaff, AZ, USA.

5. University of Washington, Seattle, WA, USA.

The fine-scale textures of rock surfaces reflect a complex interplay of geologic processes that include those related to rock formation, exposure, weathering, and erosion. Typically, such features are studied in two dimensions using forensic geological techniques from scales from those visible to the naked eye to those in a wider-area context. Rock surface metrology is an emergent technique in which extremely fine-scale textural information is measured in 3D using laboratory instruments. Here we describe its application on the surface of Mars. With the advent of sub-millimeter resolution (14-100 $\mu \mathrm{m}$ per pixel) imaging on Mars, a relatively low-cost (i.e., camera-based) approach for computing quantitative relief models (QRM) of rock surfaces has been investigated. We present preliminary assessment of 20 different martian rock surfaces using this QRM technique; the observations have implications regarding the depositional, diagenetic, and weathering/erosional history of sedimentary rocks on Mars.

The Mars Science Laboratory (MSL) Curiosity rover landed in Gale crater, Mars, to investigate a portion of the 5-km-thick stratigraphic section of largely sedimentary rocks exposed within the crater. These rocks display records of depositional and diagenetic environments thought to be $\sim 3.6$ billion years old and include fluvial conglomerates; fluvial, deltaic, and eolian sandstones; and lacustrine mudstones [1]. The Mars Hand Lens Imager (MAHLI) is a 2-megapixel Bayer pattern micro-filtered color camera with a focusable macro lens mounted on the turret at the end of Curiosity's robotic arm [2]. We used MAHLI to acquire sets of 5 overlapping frames arranged in a “+” pattern with $\sim 31 \mu \mathrm{m} /$ pixel resolution to compute QRMs at scales never before possible on natural planetary surfaces [3]. These QRM's allow analysis and interpretation of geologic surfaces at horizontal scales as fine as $100 \mu \mathrm{m}$, akin to nondestructive engineering metrology approaches. In principle, the quantitative textural information that QRMs convey for exposed rock surfaces on Mars is tied directly to the cumulative processes that formed, emplaced, and modified them. Thus, our QRM analysis of 20 different Mars rock surfaces offers a new opportunity to extract information about the depositional, diagenetic, and in situ weathering processes on Mars at spatial scales greater than $0.1 \mathrm{~mm}$, extending the array of information about the role of diagenesis and other processes on Mars from mm-scale chemistry (i.e., ChemCam on Curiosity). We generated a series of QRM datasets, starting with a high-silica sandstone known as Greenstone (Sol 1130) and continuing to our latest rock surface targets named Belle Lake (Sol 1586) and Misery (Sol 1593). We analyzed the spatial and vertical distribution of rock surface 'texture elements' at multiple scales, from $0.1 \mathrm{~mm}$ to several $\mathrm{mm}$ in an effort to unravel their geologic histories. Critical to understanding the geologic phenomena captured in the QRMs are quantitative comparisons with terrestrial reference surfaces, which is an effort presently underway using NASA non-destructive engineering facilities. In addition, we investigated the use of scale-invariant local texture classification 
methods, derived from those used for geomorphologic studies of terrestrial landscapes. These methods, known as "geomorphons", are a pattern-recognition approach to classification and mapping and were developed initially using digital topography data sampled at 5-100 m horizontal scales [4]. In our application, we extended this technique to spatial scales from $0.1 \mathrm{~mm}$ to $10 \mathrm{~mm}$ on rock surfaces, covering a typical area of $\sim 5 \mathrm{~cm} \times 3.7 \mathrm{~cm}$. Our latest work suggests that the $100 \mu \mathrm{m}$ gridded QRM's offer $\sim 30 \mu \mathrm{m}$ vertical precision for rock surfaces. Figure 1 illustrates MAHLI QRM's of four distinctive rock surfaces, including two mudstones (Belle Lake and Misery), a coarse eolian sand surface (Barby), and a laminated rock surface (Conda). For each of the QRMs illustrated using a color, hillshaded relief model (map), we have also included a micro-geomorphon computation [4] that classifies the textures into a small number of "relief elements" (ridges, troughs, smooth areas, etc.) as a means of allowing for objective inter-comparison. On this basis, there appear to be distinctive martian rock surface texture "signatures" apparent in the QRMs and identifiable in the micro-geomorphon maps. Cracked and vein-riddled surfaces such as Misery are distinct from otherwise modified surfaces such as those at Belle Lake. These preliminary results provide encouragement that this method will contribute to our understanding of habitability of Mars, as gleaned from the rock record.

\section{References:}

[1] J Grotzinger et al., Science 350 (2015), doi:10.1126/science.aac7575.

[2] R Yingst et al., Geosci. Instrum. Method. Data Syst. 5 (2016), doi:10.5194/gi-5-205-2016.

[3] J Garvin et al., Lunar Planetary Science Conference 47 (2016), \# 2834.

[4] J Jasiewicz and T Stepinski, Geomorphology 182 (2013), 147-156.

[5] The authors acknowledge support from the MSL Project, courtesy NASA HQ and JPL.

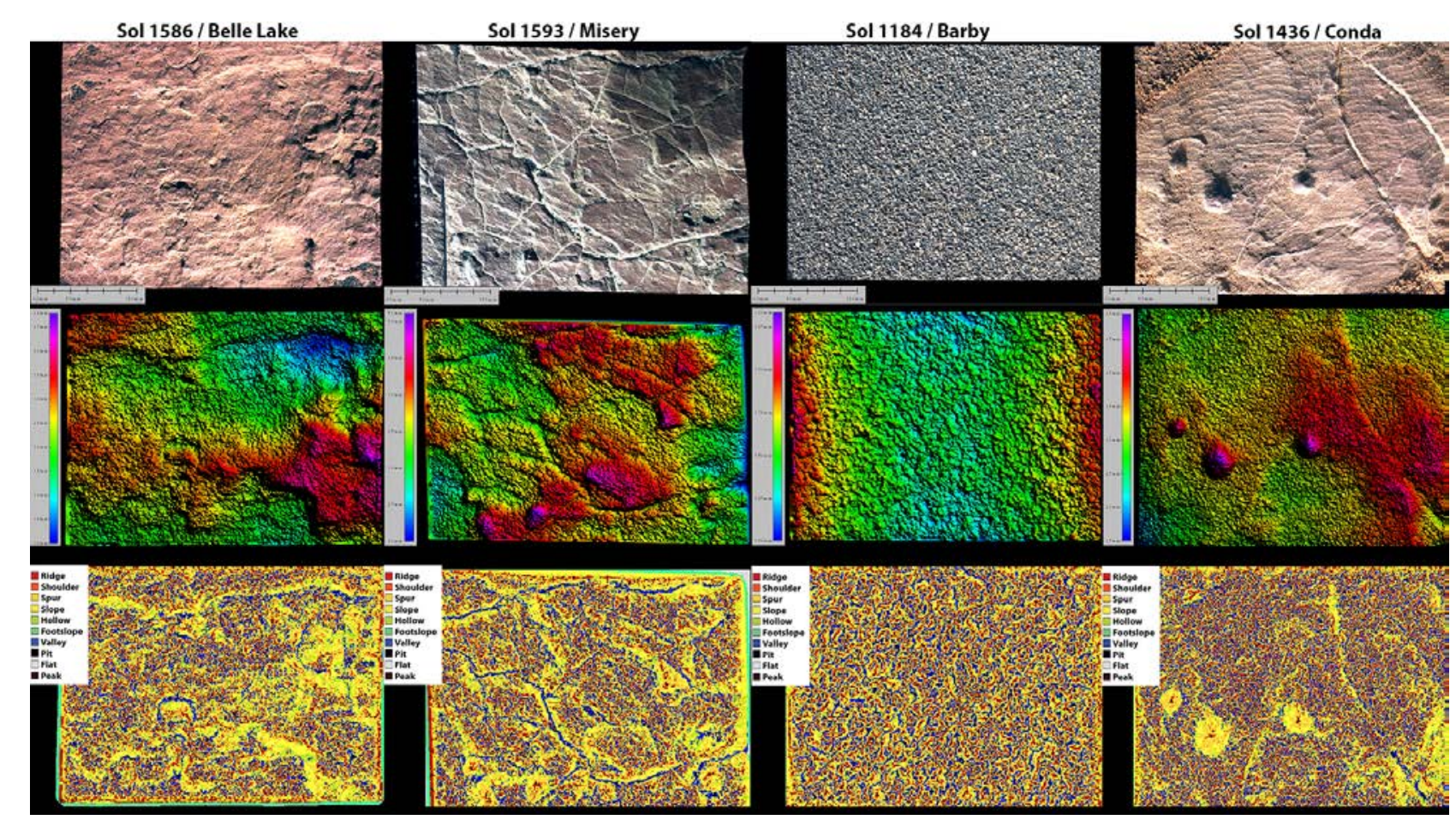

Figure 1. Example of Mars Curiosity rover MAHLI Quantitative Relief Models (QRM) of example rock surfaces on Mars (i.e., field of view $\sim 4.9 \mathrm{~cm}$ x $3.7 \mathrm{~cm}$ with QRM ground scale distance of 100 $\mu \mathrm{m})$. Martian rock surface examples illustrated in terms of $30 \mu \mathrm{m}$ MAHLI images (top row), QRM maps (middle row), and micro-geomorphon classifications (lower row). See text for details. 